\title{
Kinematic Design of a Two Contact Points Haptic Interface for the Thumb and Index Fingers of the Hand
}

Francesco Simoncini Massimo Bergamasco Fabio Salsedo

\author{
Scuola Superiore Sant'Anna, \\ PERCRO, \\ Polo Sant'Anna Valdera, \\ Viale Rinaldo Piaggio 34, Pontedera, \\ Pisa 56025, Italy
}

This paper presents an integrated approach to the kinematic design of a portable haptic interface for the thumb and index fingers of the hand. The kinematics of the haptic interface was selected on the basis of constructive reasons, design constraints, and usability issues, and in order to guarantee the best level of performance with the lowest encumbrance and weight over the workspace of the hand. The kinematic dimensioning was the result of a multi-objective optimization of several performance parameters, such as minimum required torque at actuators and maximum reachable workspace, with the simultaneous fulfillment of design constraints, such as satisfactory mechanical stiffness at the end effector, global kinematic isotropy over the workspace, and limited bulk of the device. A geometric interpretation of singularities based on screw theory was formulated to point out both hand postures and movements associated with weaker performance. The results of the paper were used to build the prototype of a new portable haptic interface with two contact points, whose main design features are also specifically presented. [DOI: $10.1115 / 1.2712219]$

\section{Introduction}

In one class of haptic devices, robotic systems are employed to physically grasp and manipulate digital objects in virtual environments. They are usually composed of several actuated mechanical limbs that can track and independently apply a force to one or more fingertips, in relation to the contact status of the simulation [1].

According to studies conducted in biomechanics [2], the particular nature of the joints of the anatomical complex of human hand and wrist can provide a huge mobility to the tip of each finger. In fact as a distinctive trait of the human gender, the thumb can work in opposition with each finger of the hand, allowing dexter grasping and fine manipulation of objects. Psychoperceptual studies have confirmed that multiple points of contact on the operator's hand are necessary to simulate tasks such as grasping or shape recognition of objects in virtual worlds [3-5]. However the design of mechanisms allowing a multipoint haptic interaction still poses several design and technological challenges.

Light and stiff mechanisms with the same workspace and kinematic dexterity of human fingers have been investigated both in the design of anthropomorphic robot hands [6-8], where several interesting solutions have been proposed in terms of cable driven actuation, and of hand exoskeletons. Hand exoskeletons, like the LRP hand master [9], the PERCRO HFF [10], and other ones [11-13], are a class of haptic devices for the hand characterized by a dorsal structure, reproducing the kinematic chain of the finger on which they are put, that can apply forces over several points of the fingers. Remote centers of rotation or originally specifically devised mechanisms are usually used in hand exoskeletons to avoid mechanical interference with fingers.

Typically, as their main limitation, hand exoskeletons cannot

Contributed by the Mechanisms and Robotics Committee of ASME for publication in the Journal of Mechanical Design. Manuscript received December 16, 2005 final manuscript received May 25, 2006. Review conducted by Pierre M. Larochelle. Paper presented at the 2002 ASME Design Engineering Technical Conferences \& Computers and Information in Engineering Conference (DETC2002), Montreal, Quebec, Canada, September 29-October 2, 2002. apply forces with arbitrary direction at the level of the fingertip, but only forces directed normally to each phalanx, so that only simulations of grasping of objects are allowed.

This paper presents a new portable haptic interface for the thumb and index fingers of the hand, that overcomes these limitations. The results of the paper were used to build a prototype of a portable haptic interface with two contact points [14], whose main design features are presented. The kinematic design of the serial mechanism of each limb composing the device was optimized to track the motion of index and thumb fingertips, generated by the combined movements of the phalanx articulations of each finger and the wrist joint of the human hand.

While the kinematics of the limb mechanism was selected on the basis of constructive, design, and usability issues, the kinematic dimensions of the mechanism were optimized as the smallest ones ensuring a significant performance all over the workspace covered by the hand, that can be equivalently thought of as a mechanism with a higher mobility. The final dimensions were determined through multiobjective optimization of several performance indexes, such as minimum required torque at the actuators and maximum reachable workspace, with the fulfillment of design constraints, such as satisfactory mechanical stiffness at the end effector, global kinematic isotropy over the workspace, and limited bulk of the device.

The optimization procedure was formulated by taking into account the location of singularities of the mechanism within the workspace and using a geometric interpretation based on screw theory, to point out both critical hand postures and movements.

The rest of this paper is organized as follows. The main guidelines followed in the design of one limb mechanism are presented in Sec. 2. A kinematic analysis of the mechanisms functional to the performance and to the ergonomic evaluation is reported in Sec. 3, including a kinematic model of the human wrist and hand complex, while the optimization strategy followed in the kinematic dimensioning of the mechanism is reported in Sec. 4. Discussion on results of the optimization procedure and on main findings are reported in Sec. 5, and finally conclusions of the study are drawn in Sec. 6. 


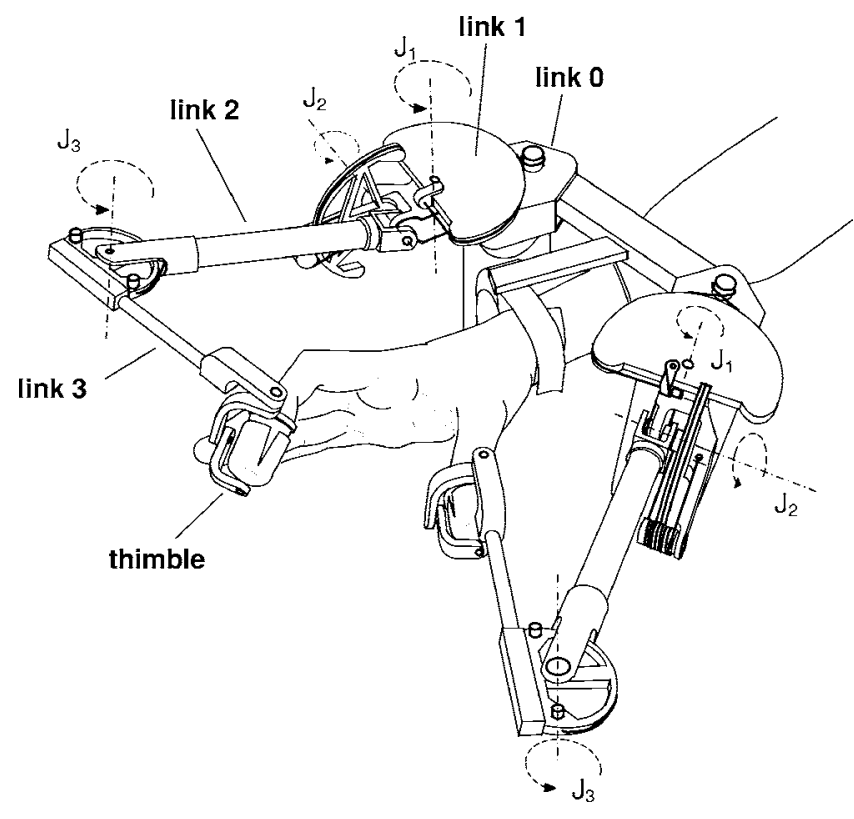

Fig. 1 A general scheme of the mechanism

\section{Mechanical Design of the Limb Mechanism}

A scheme of the adopted kinematic solution is shown in Fig. 1. The mechanism for each limb is chosen in order to satisfy the following constraints [15]:

1. The link 0 is fixed on the forearm (either supported by an actuated or passive device balancing only its weight);

2. It is composed of two serial limbs, herein after called the finger mechanisms, each one with three actuated degree of freedoms (DOFs) and four links, reaching the thumb and index fingertips;

3. The two finger mechanisms can track any movement of the thumb and index fingertips, including hand rotations generated by the wrist;

4. The actuation of joints is implemented by means of a tendon drive, and all motors are located on link 0 ;

5. All kinematic pairs are implemented through rotational joints; and

6. Only the first three joints $J_{1}, J_{2}, J_{3}$ of each limb are actuated.

As to the first point of this list, in the typical configuration the support for the weight of the device is provided through an actuated force-feedback arm exoskeleton [16], used in combination with this haptic interface for the hand, that can guarantee the full mobility of the arm.

A thimble mounted on a spherical passive joint is placed at the end of each finger mechanism to reach the user's fingertip with any orientation, where the force feedback is applied. In order to decrease the weight of the moving masses and enhance the backdrivability of the system, a bidirectional tendon driven [17] is adopted to transmit the torques from each actuator to the actuated joint, implementing a remote actuation scheme. The kinematics of each finger is chosen to satisfy the constraints of the routing of the tendon drive, requiring that driven and idle pulleys of two consecutive joint axes should lie in the same plane.

Figure 2 shows a side and a slanted view of each finger mechanism, and the scheme of the tendon routing adopted for each transmission [18]. The motor pulleys $(1,2,3)$ are placed on link 0 and are directly connected to the motors $(m 1, m 2, m 3)$. Transmission 1 starts from motor axis $1(a 1)$ and connects the motor pulley (1) to the capstan (4) placed on joint $1(j 1)$, that is fixed to link 1. Transmission 2 starts from the motor pulley (2) on the axis $a 2$, it is routed over the first joint through idle pulleys both in the forward $(5,6)$ and backward $\left(5^{\prime}, 6^{\prime}\right)$ direction and then reaches the cable speed reducer $(7,8)$ where the actuation torque is delivered

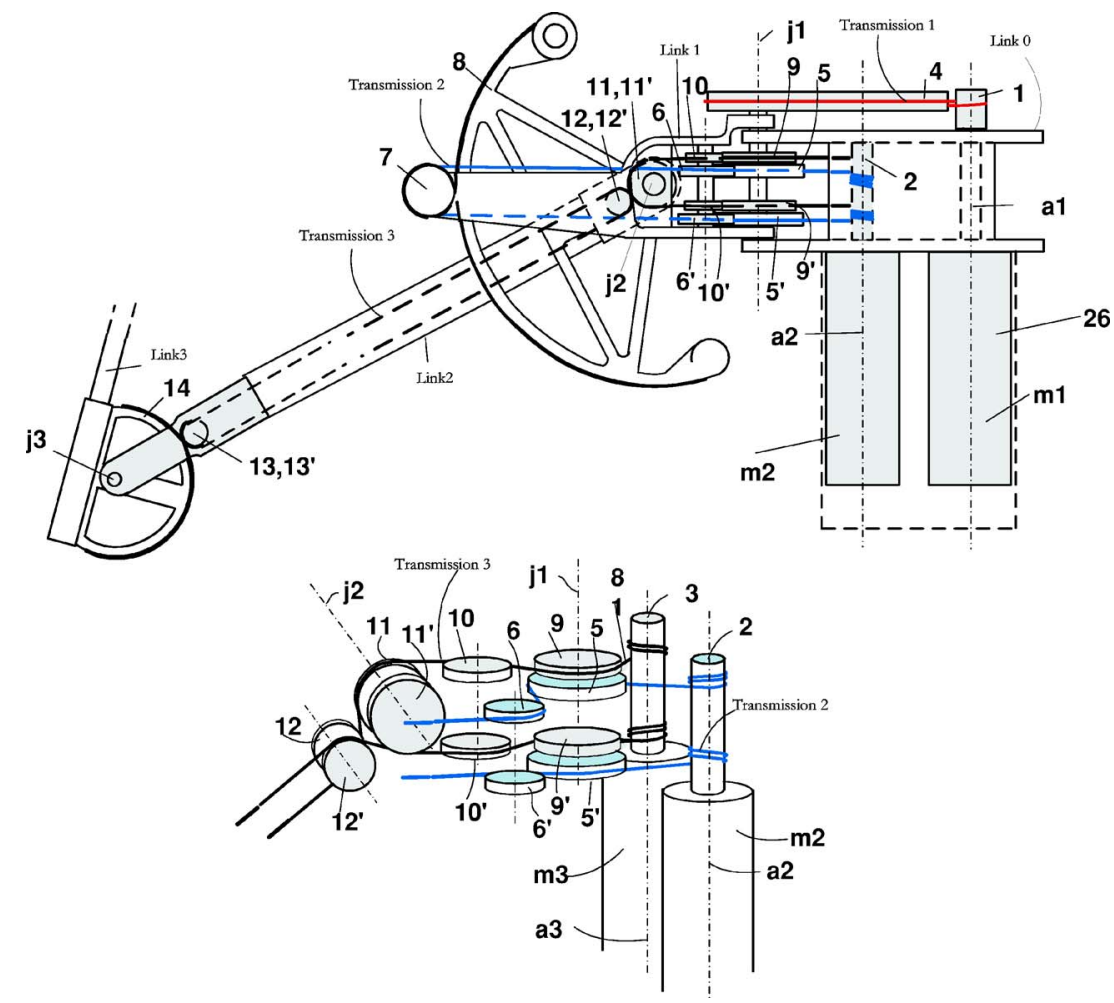

Fig. 2 Side (top) and slanted (bottom) view of the tendon transmission [18] 


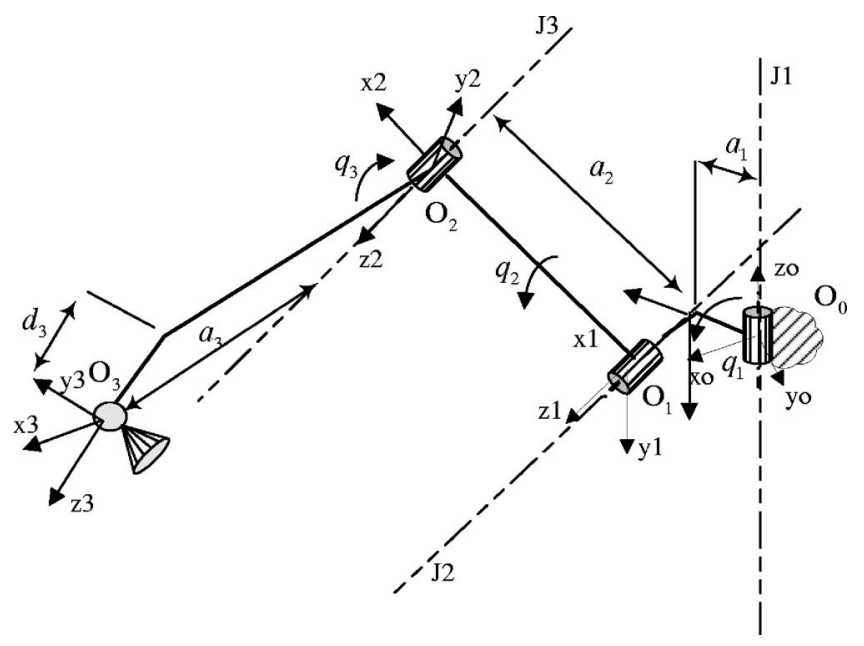

Fig. 3 The kinematics of each finger mechanism

to the capstan (8) fixed to link 1. Transmission 3 begins at axis a3, and is routed through the first joint and the second joint respectively by the group of idle pulleys $\left(9,10,9^{\prime}, 10^{\prime}\right)$ and $\left(11,12,11^{\prime}, 12^{\prime}\right)$. Then the cable runs inside the cover of link 2 and reaches the driven pulley (14) through the idle pulleys $\left(13,13^{\prime}\right)$ where the actuation torque is delivered to link 3 .

\section{Kinematic Analysis}

The resulting kinematics of each finger mechanism, shown in Fig. 3, is composed of three mutual orthogonal $R$ joints.

Denavit-Hartenberg (DH) parameters $d_{i}, a_{i}, \alpha_{i}, \theta_{i}[19]$ are used to model the kinematics of the system and a reference frame $O_{i} x_{i} y_{i} z_{i}$ can be associated to each link according to the DH convention, as shown in Fig. 3.

The offsets $d_{1}, d_{3}, a_{1}$ are kept to the smallest possible values that satisfy the constructive constraints imposed by the mechanical design of the transmission system. Their lengths are much smaller than the link lengths $a_{2}$ and $a_{3}$, so that their values can be neglected for a first approximation kinematic analysis of the system.

3.1 Kineto-Static Analysis of the Finger Mechanism. The position of the two robotic fingers on the forearm is determined by the kinematic parameters shown in Fig. 4 . The parameters $L_{1 i}, L_{2 i}$ and $L_{1 t}, L_{2 t}$ set the position of the link 0 along the forearm, respectively, for the index mechanism and for the thumb mechanism, and hereinafter will be called $L_{1}, L_{2}$ where they are not referred to a particular finger. $L_{1}$ is the distance along the forearm axis between the base coordinate system of a finger mechanism and the base reference frame of the wrist.

The angles $\alpha_{i}, \alpha_{t}$ represents the orientation of link 1 with respect to the forearm axis in the reference configuration, respectively, for the index and thumb finger.

The performance of the mechanism over its workspace can be analyzed as a function of the reachable workspace by the human hand. A kinematic model of the hand has been specifically developed for this purpose.

3.1.1 Kinematic Model of Human Hand. According to Buchholz et al. [2], the kinematic model of the hand is developed assuming negligible compliance of both cartilages and tendons and replacing the complex real kinematic pairs of human joints with rotational joints. As shown in Fig. 5, the hand is composed of a wrist articulation, modeled with 2 DOFs, from which two serial open chains depart, respectively, with 6DOF and 4DOF, modeling the thumb and index fingers. The hand kinematic model was used to generate the exact position spanned by the index and thumb

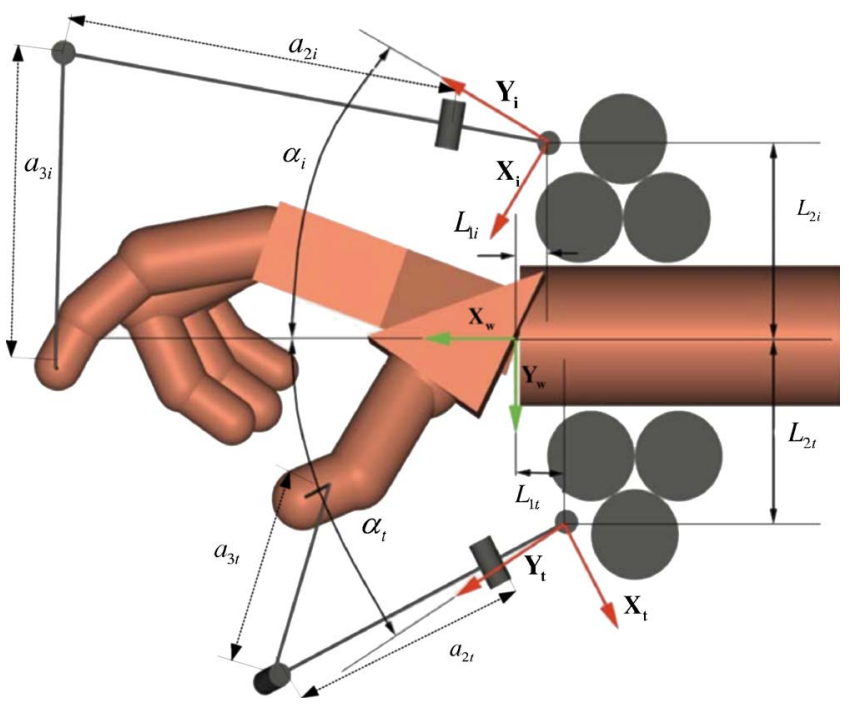

Fig. 4 Parameters used for describing the positioning of the system on the forearm

fingertips for regular increases of each joint angle of fingers and wrist. These positions were inputted to the inverse kinematic model of each finger mechanism, in order to study their performance in the workspace reachable by the hand.

The kinematic parameters for this model (number and arrangement of the DOFs, limitation of joint angles, mean lengths of the phalanxes, and relative proportion between them) were estimated from hand anatomy, both through measurements taken by hand photographs in different projections and through data existing in the literature [20]. The design of the mechanism was optimized for the largest likely dimensions of hand segments, according to the anthropometric measurements reported in standardized tables [21]. The lengths assumed for the optimization analysis are reported in Tables 1-3, expressed as Denavit-Hartenberg parameters [19], measured in $\mathrm{mm}$.

The geometric interpretation of the singularities and the results of the kinematic optimization, presented in the next sections, has allowed us to characterize the sensitivity of the design to hand parameters: in particular larger values of the hand dimensions reduce the extension-flexion wrist range $\alpha_{W}$, with a greater re-

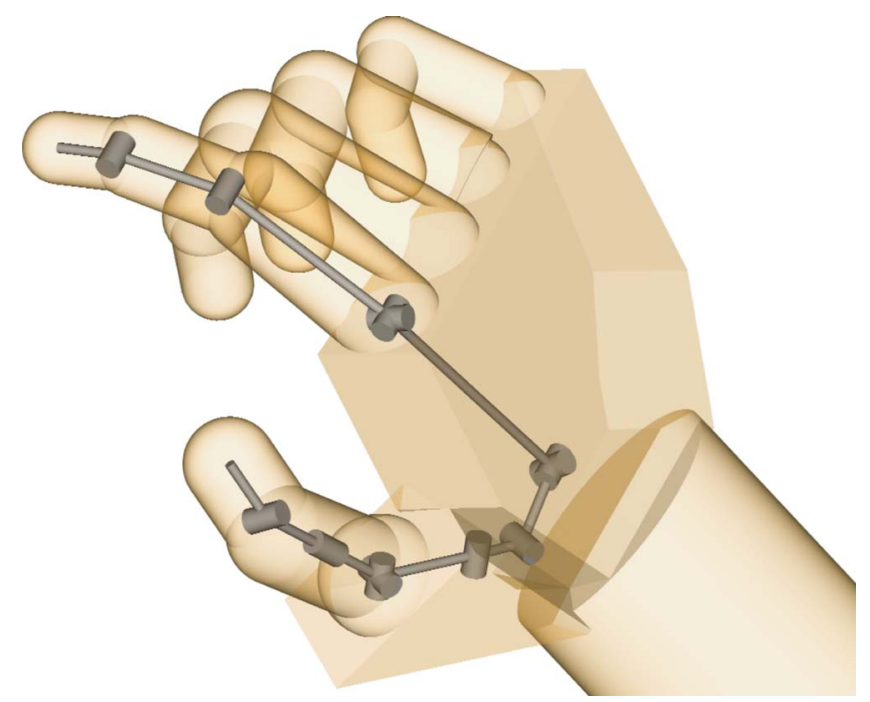

Fig. 5 The kinematic model of the hand 
Table 1 DH nominal parameters of the thumb kinematic model

\begin{tabular}{cccc}
\hline \hline$J$ & $a_{i}$ & $d_{i}$ & $\alpha_{i}$ \\
\hline $1_{t}$ & 10.6 & 0 & $\pi / 2$ \\
$2_{t}$ & 32.6 & 0 & 0 \\
$3_{t}$ & 0 & 0 & $-\pi / 2$ \\
$4_{t}$ & 0 & 0 & $-\pi / 2$ \\
$5_{t}$ & 0 & 29.9 & $-\pi / 2$ \\
$6_{t}$ & 23 & 0 & 0 \\
\hline \hline
\end{tabular}

striction of the thumb workspace, whose mobility is constrained by the mechanism to a greater extent than the index.

3.2 A Geometric Interpretation of Kinematic Singularities. The kinematic singularities of the mechanism, as moved by the hand, represent the most limiting factor of performance for each finger mechanism, that can be easily analyzed in a geometric way by screw theory [22]

With reference to Fig. 3, the equations governing the kinetostatics behavior of each finger are given by

$$
\mathbf{v}=J\left(\begin{array}{c}
\dot{q}_{1} \\
\dot{q}_{2} \\
\dot{q}_{3}
\end{array}\right) \quad\left(\begin{array}{c}
\tau_{1} \\
\tau_{2} \\
\tau_{3}
\end{array}\right)=J^{T} \mathbf{f}
$$

The vector $\mathbf{v}$ and $\mathbf{f}$ are, respectively, the velocity and the force applied at the end effector $O_{3}$, while $q_{i}$ and $\tau_{i}$ represent, respectively, position and torque of joint $i$. The geometric analysis is not affected by the choice of the frame of reference, but for sake of clarity all the vectors will be assumed to be represented in the frame $O_{3} x_{3} y_{3} z_{3}$ (see Fig. 3), adopting the point $O_{3}$ as pole of representation for all the screws represented in axial coordinates.

The general expression of the Jacobian of the system can be expressed as a function of the screw $\$_{i-1}^{i}$ associated to each joint

$$
\$_{i-1}^{i}=\left(\begin{array}{c}
\mathbf{s}_{\mathbf{i}} \\
\mathbf{s}_{\mathbf{o}, \mathbf{i}}
\end{array}\right)=\left(\begin{array}{c}
\mathbf{z}_{\mathbf{i}-\mathbf{1}} \\
\left(\mathbf{o}_{\mathbf{i}-\mathbf{1}}-\mathbf{o}_{\mathbf{3}}\right) \wedge \mathbf{z}_{\mathbf{i}-\mathbf{1}}
\end{array}\right)
$$

Since the spherical kinematic pair is centered at $\mathrm{O}_{3}$, only forces f of arbitrary direction and magnitude centered at $\mathrm{O}_{3}$ can be transmitted by each actuated finger mechanism and so can be represented by a wrench of zero pitch through $\mathrm{O}_{3}$

$$
W_{F}=\left(\begin{array}{l}
\mathbf{f} \\
\mathbf{0}
\end{array}\right)
$$

At singular configurations, the mechanism looses its capacity of controlling the actuation force $\mathbf{f}$ applied at the end point $\mathrm{O}_{3}$ along one direction, and consequently the ability to simulate contacts with a virtual wall with an arbitrary orientation in space. In these

Table 2 DH nominal parameters of the index kinematic model

\begin{tabular}{cccc}
\hline \hline$J$ & $a_{i}$ & $d_{i}$ & $\alpha_{i}$ \\
\hline $1_{i}$ & 0 & 0 & $\pi / 2$ \\
$2_{i}$ & 40.3 & 0 & 0 \\
$3_{i}$ & 23.3 & 0 & 0 \\
$4_{i}$ & 17.8 & 0 & 0 \\
\hline \hline
\end{tabular}

Table 3 DH nominal parameters of the wrist kinematic model

\begin{tabular}{cccc}
\hline \hline$J$ & $a_{i}$ & $d_{i}$ & $\alpha_{i}$ \\
\hline $1_{w}$ & 0 & 0 & $\pi / 2$ \\
$2_{w}$ & 0 & 0 & 0 \\
\hline \hline
\end{tabular}

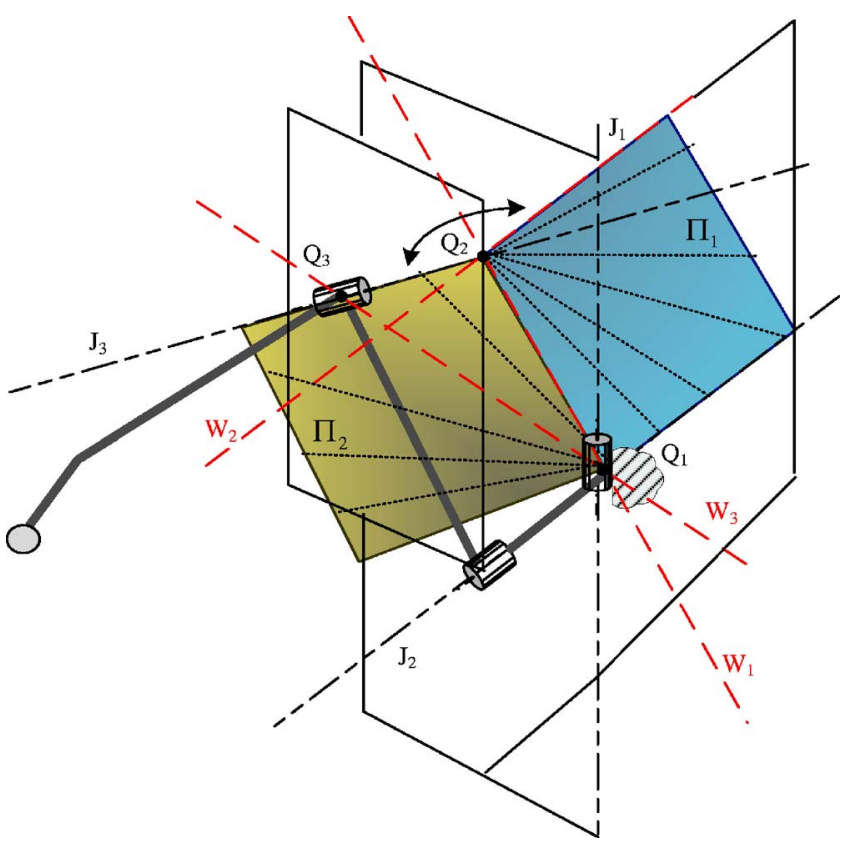

Fig. 6 Geometric interpretation of singularities for the case $a_{1} \cong 0$

configurations there exists a wrench $W_{F}$ that belongs to the system of wrenches $\mathcal{W}$ satisfying the reciprocity condition expressed by the dual product $\otimes[22]$

$$
\$_{i-1}^{i} \otimes W=\mathbf{w}_{\mathbf{o}} \cdot \mathbf{s}_{\mathbf{i}-\mathbf{1}}^{\mathbf{i}}+\mathbf{w} \cdot \mathbf{s}_{\mathbf{0}, \mathbf{i}}^{\mathbf{i}-\mathbf{1}}=0 \quad \forall i=1,2,3
$$

This wrench system $\mathcal{W}$ can be found by means of line geometry and in particular a straight geometric procedure can be devised in the case that the first two joints $J 1$ and $J 2$ are incident, or equivalently that $a_{1}=0$, as shown in Fig. 6. This constitutes a close approximation of the kinematics of the real system, in reason of the small value of $a_{1}$. In this case the system $\mathcal{W}$ is generated by the linear combination of three wrenches of zero pitch (pure forces), belonging to a degenerate regulus of an hyperboloid, made of two planar pencils with a common wrench [23].

Define the following datum points and planes

- Take the point $Q_{1}$ at the intersection of axes $J_{1}$ and $J_{2}$

$$
Q_{1}: \quad J_{1} \cap J_{2}
$$

- Take the plane $\Pi_{1}$ through the axes $J_{1}$ and $J_{2}$

$$
\Pi_{1}: \operatorname{Pl}\left(J_{1}, J_{2}\right)
$$

- Take the point $Q_{2}$ at the intersection of axis $J_{3}$ with the plane $\Pi_{1}$

$$
Q_{1}: \quad \Pi_{1} \cap J_{3}
$$

- Take another point $Q_{3}$ on the axis $J_{3}$, different from $Q_{2}$

$$
Q_{3}: \quad \in J_{3}, \neq Q_{2}
$$

- $\quad$ Take the plane $\Pi_{2}$ through all the points $Q_{i}, i=1,2,3$

$$
\Pi_{2}: \quad \operatorname{Pl}\left(Q_{1}, Q_{2}, Q_{3}\right)
$$

The three generators $W_{i}$ of the reciprocal wrench system $\mathcal{W}$ can then be defined as follows:

- $W_{1}$ is a wrench of zero pitch aligned along $\overline{Q_{1} Q_{2}}$;

- $W_{2}$ is a wrench of zero pitch through $Q_{2}$ and parallel to $J_{2}$; and

- $W_{3}$ is a wrench of zero pitch aligned along $\overline{Q_{1} Q_{3}}$. 


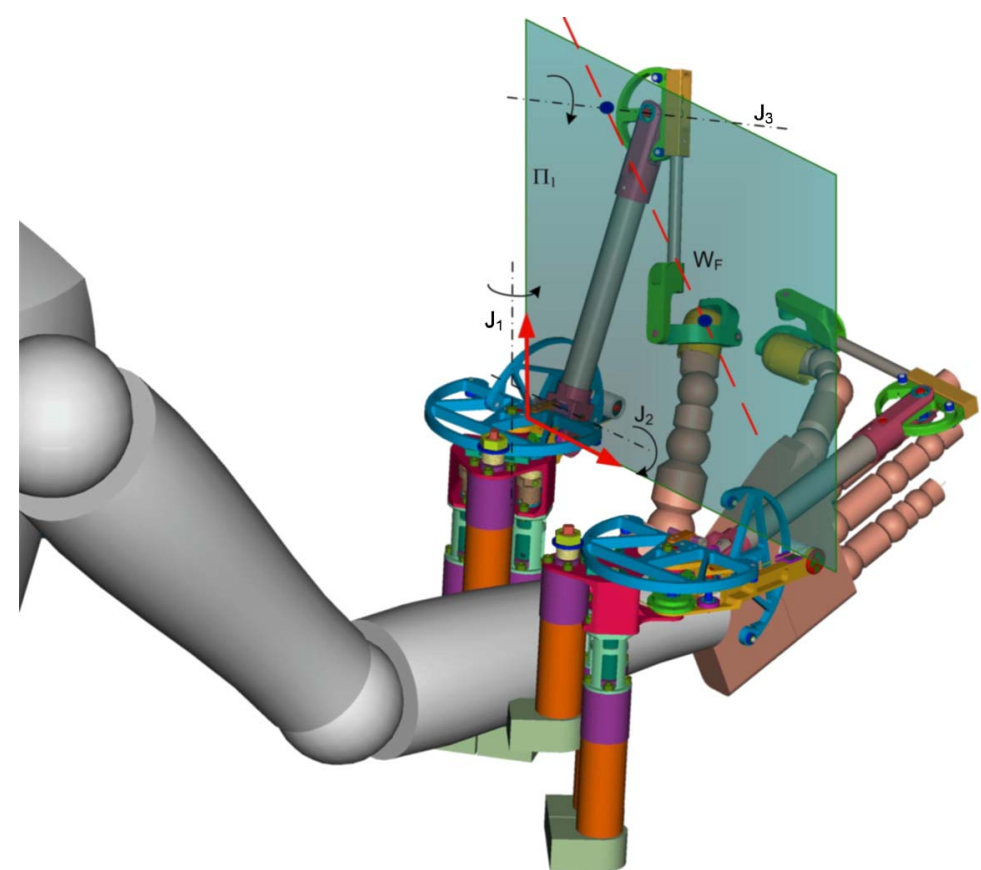

Fig. 7 The CAD model of the system in a singularity condition

As shown in Fig. 6, the linear combination of these three generators $W_{i}$ form two planar pencils of wrenches of zero pitch with a common generator, aligned along the direction $\overline{Q_{1} Q_{2}}$, and lying in the two planes $\Pi_{1}, \Pi_{2}$. All the lines belonging to the two planar pencils satisfy Eq. (4), since have the property of intersecting all the joint axes $J_{i}$.

After having found the two planes $\Pi_{1}, \Pi_{2}$ in an assigned configuration for a given set of values of $q_{1}, q_{2}$, we can observe that there always exists a rotation angle $q_{3}$ around $\$_{3}^{2}$ that brings the end effector $\mathrm{O}_{3}$ into one of the two planes, without changing the geometry of the reciprocal system $\mathcal{W}$.

When such a posture, as the one shown in Fig. 7, is assumed by the mechanism, the $3 \times 3$ Jacobian $J$ of Eq. (1) becomes singular and the mechanism looses the capability of actuating a force along the wrench $W_{F} \in \mathcal{W}$ through $O_{3}$, since a force applied along such a direction intersect all the joint axes $\$_{i-1}^{i}$.

This geometric representation provides a useful interpretation of the kinematic configurations in which the mechanism is close to singularities: for instance when a postures with the metacarpal joint of the thumb extended and the wrist is flexed is reached, like the one shown in Fig. 7, a remarkable reduction of the performance of the finger mechanism is attained.

When $a_{1} \neq 0$, a nondegenerate regulus of wrenches of zero pitch is formed, that for small values of $a_{1}$ can still be approximated by the two the two planar pencils through $\Pi_{1}$ and $\Pi_{2}$.

A wood mockup of the system confirmed that the main critical postures were produced by an excessive extension of the metacarpal joint of the thumb associated to a simultaneous flexion of the wrist.

\section{Kinematic Optimization and Analysis of Perfor- mance}

The optimal kinematic design of the mechanism is the one that can guarantee the best performance over the workspace of the hand with the lowest associated encumbrance and weight. While the weight is highly related to the size of the adopted motors, the encumbrance is related to the kinematic dimensioning of the mechanism. On the other hand, the performance of the system is characterized by the isotropy of behavior over the workspace and capability of exerting forces and displaying mechanical stiffness at the end effector. Overall four parameters of performance were selected as the most significant to characterize the performance of the system:

- $\mathbf{W S}=$ size of the reachable workspace;

- $\mathbf{G I I = g l o b a l ~ k i n e m a t i c ~ i s o t r o p y , ~ e x p r e s s e d ~ b y ~ a ~ g l o b a l ~ i s o t - ~}$ ropy index;

- $\mathbf{R T}=$ maximum required torque at actuators; and

- $\mathbf{M S}=$ mechanical stiffness displayed at the end effector.

The first two parameters WS and GII are clearly related with the size of the mechanism, while the other two ones RT and MS are inversely related. It is clear so that a multiobjective optimization approach is required to formulate and correctly address the design problem, and that the resultant optimal design will result in a tradeoff between the different needs expressed by the above four parameters of performance.

For each finger mechanism, $a_{2}, a_{3}$, and $L_{1}$, shown in Fig. 4, constitute the kinematic parameters determining the performance of the device. The optimization analysis assumed $L_{1}$ as the independent parameter corresponding to different sizes of the device, and searched for the optimal analytical relationship for the other two performance parameters as $a_{2}\left(L_{1}\right)$ and $a_{3}\left(L_{1}\right)$. As to the performance parameters, the following definitions were adopted.

4.1 Size of the Reachable Workspace. The finger mechanism should be capable of tracking at the best the movements of the thumb and index fingers, including the ones generated by the rotations of the wrist. The workspace generated by the human hand can be described in terms of the motion generated by the finger and wrist joints and computed through the analytical kinematic model of the wrist/hand complex.

The wrist motion was approximated by two rotations around two orthogonal axes placed at the base of the kinematic chain of the hand, respectively, in the frontal plane and in the sagittal plane, associated to the abduction-adduction and flexionextension of the wrist. The flexion-extension of the wrist, shown in Fig. 8, is characterized by a rotation of an angle $\alpha_{w}$, and covers a wider angular range than the abduction-adduction; it represents a more critical motion to be tracked by the finger mechanisms, since it brings the mechanism closer to singularity configurations, 


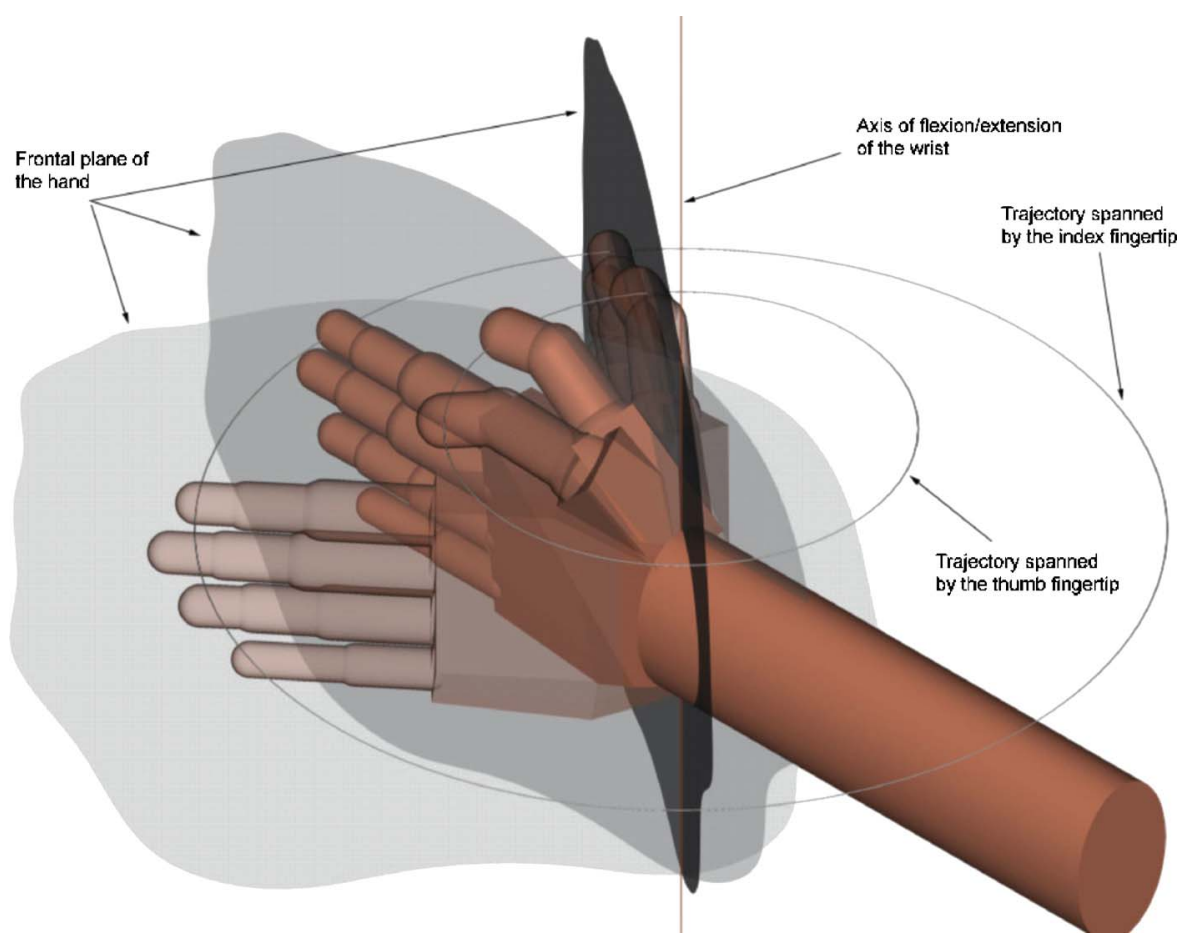

Fig. 8 The movement of flexion-extension of the wrist associated to the angle $\alpha_{w}$

i.e., moving the end point toward the plane $\Pi_{1}$.

While the wrist movements contribute to considerably increase the workspace spanned by each finger, they influence to a lesser extent the fingers ability of manipulating and grasping, that is mainly determined by the movements generated by the finger joints.

On the basis of the above considerations, the amount of allowable angular flexion-extension $\alpha_{w}$, measured in degrees, was chosen as the main variable to parameterize different sizes of the workspace, since it represents at the same time the most critical movement limiting the performance of the mechanism and determining the greatest variations of spanned workspace, without changing the capability of dexterous manipulation. For each value of $\alpha_{w}$, the mechanisms included in the analysis were required to fully cover the range of motion generated by the wrist abductionadduction and finger joints.

4.2 Global Kinematic Isotropy. The ability of the finger mechanisms to follow the movement of the fingertips with good kinematic properties was measured by means of a index of global kinematic isotropy over the workspace. The global isotropy index (GII) [24] is defined as the ratio between the maximum of the largest semi-axes and the minimum of the smallest semi-axes of the manipulability ellipsoids over the entire workspace

$$
\mathrm{GII}=\frac{\max _{\mathcal{W}_{h}}\left[\sigma_{\max }\left(J J^{T}\right)\right]}{\min _{\mathcal{W}_{h}}\left[\sigma_{\min }\left(J J^{T}\right)\right]}
$$

A GII of 1 is achieved when the manipulability ellipsoids are spheres with equal radius in all the points of the workspace. Acceptable configurations were defined as the ones with GII not exceeding the value of $\mathrm{GII}_{\text {target }}$.

4.3 Maximum Torque at Actuators. The bulk and weight of the actuators is strictly dependent on the maximum required torques. Motor torques $\tau_{m, i}$ are related to joint torques $\tau_{i}$ by a triangular transmission matrix $T[17,25]$ depending on the radii of the tendon drive pulleys, that can be expressed as

$$
\boldsymbol{\tau}=\left(\begin{array}{ccc}
r_{11} & r_{21} & r_{31} \\
0 & r_{22} & r_{32} \\
0 & 0 & r_{33}
\end{array}\right) \boldsymbol{\tau}_{\mathbf{m}}=T \boldsymbol{\tau}_{\mathbf{m}}
$$

where the parameter $r_{i j}$ indicates the radius of a pulley at joint $j$ belonging to transmission $i$.

For each choice of the parameters $a_{2}, a_{3}$, and $L_{1}$, that the maximum torque required $\overline{\tau_{m, i}}(\mathbf{p})$ for each actuator to exert at point $\mathbf{p}$ a force $\mathbf{f}$ of $1 \mathrm{~N}$ directed along an arbitrary direction can be computed.

When the end-effector applied forces are constrained to lie on a sphere of unit diameter, by the relationship

$$
\mathbf{f}^{T} \mathbf{f}=1
$$

the required actuator torques $\tau_{m, i}(\mathbf{p})$ span the surface of an ellipsoid. In fact, by using the second of Eqs. (1) and defining the matrix $A=T^{T}\left(J^{T} J\right)^{-1} T$, the constraint Eq. (12) can be put in the form

$$
\boldsymbol{\tau}_{\mathbf{m}}(\mathbf{p})^{T} A \boldsymbol{\tau}_{\mathbf{m}}(\mathbf{p})-1=0
$$

The extremal values of $\tau_{m, i}(\mathbf{p})=\boldsymbol{\tau}_{\mathbf{m}}(\mathbf{p})^{T} \mathbf{u}_{\mathbf{i}}$, where $\mathbf{u}_{\mathbf{i}}, \quad(i$ $=1,2,3)$ are the main versors associated to the Cartesian frame of reference adopted for the representation in $\mathcal{R}^{3}$, can be obtained by maximizing the expression Eq. (13) with the constraint Eq. (12). This optimization problem is equivalent to maximize the Lagrange function $\mathcal{L}$ at each point $\mathbf{p}$

$$
\mathcal{L}(\mathbf{p})=\boldsymbol{\tau}_{\mathbf{m}}^{T} \mathbf{u}_{i}-\lambda\left(\boldsymbol{\tau}_{\mathrm{m}}^{T} A \boldsymbol{\tau}_{\mathbf{m}}-1\right)
$$

where $\lambda$ is a scalar Lagrange multiplier.

The solution to the above maximization problem is

$$
\overline{\tau_{m, i}}(\mathbf{p})=\frac{1}{\sqrt{\mathbf{u}_{\mathbf{i}}^{T} A^{-T} \mathbf{u}_{\mathbf{i}}}} A^{-1} \mathbf{u}_{\mathbf{i}} \cdot \mathbf{u}_{\mathbf{i}}
$$

The maximum required torque at actuators can so be computed as the maximum torque over all the points $\mathbf{p} \in \mathcal{W}_{h}$ of the hand workspace 


$$
\overline{\tau_{m}}=\max _{\mathbf{p} \in \mathcal{W}_{h}}\left[\max _{i=1: 3} \overline{\tau_{m, i}}(\mathbf{p})\right]
$$

Its value was evaluated through a numerical evaluation of $\overline{\tau_{m, i}}$ on a grid of $8^{6}$ control points covering the whole workspace $\mathcal{W}_{h}$.

4.4 Mechanical Stiffness Displayed at the End Effector. The mechanical stiffness perceived at the end point provides the goodness of the device to simulate virtual walls [26].

Most of the mechanical compliance of the system can be considered as due to the elasticity of the adopted tendon transmission system. For this reason the minimum stiffness was evaluated as the inverse of the maximum compliance $\bar{c}$ at the end effector, due to the tendons elasticity, when subjected to a force generically oriented and satisfying relation (12). This definition guarantees that in every reachable point, the mechanism is able to simulate the contact with a surface whose stiffness is at least equal to $\underline{k}$ $=1 / \bar{c}$.

The stiffness matrix of the tendon transmission $K$ (or its inverse, the compliance matrix $C$ ) allows to obtain the motors displacements $\boldsymbol{\delta} \mathbf{q}$ due to tendons stretching under load as a function of applied torques $\tau$

$$
\delta \mathbf{q}=K^{-1} \boldsymbol{\tau}=C \boldsymbol{\tau}
$$

where for a tendon transmission $C$ is depending only on the length $l_{i}$ and section $A_{i}$ of the cable branches for each transmission $i$ and by the equivalent Young modulus $E$, corresponding to the elastic properties of the adopted steel cable

$$
C=\left(\begin{array}{ccc}
\frac{l_{1}}{E A_{1}} & 0 & 0 \\
0 & \frac{l_{2}}{E A_{1}} & 0 \\
0 & 0 & \frac{l_{3}}{E A_{1}}
\end{array}\right)
$$

By using Eqs. (17) and (1) and defining $\hat{C}=J C J^{T}$, Eq. (12) becomes

$$
\boldsymbol{\delta} \mathbf{x}^{T}\left(\hat{C} \hat{C}^{T}\right)^{-1} \boldsymbol{\delta} \mathbf{x}=1
$$

where $\delta \mathbf{x}$ is the end-effector displacement due to the tendons stretching under load, when the actuators are locked.

The maximum compliance is then given by

$$
\bar{c}=\max _{\mathcal{W}_{h}}\left\{\max _{i}\left[\sqrt{\sigma_{i}\left(\hat{C} \hat{C}^{T}\right)}\right]\right\}
$$

where $\sigma_{i}\left(\hat{C} \hat{C}^{T}\right)$ is an eigenvalue of the core of the quadratic form defined in Eq. (19).

4.4.1 The Optimization Procedure. Taking into account all the above definitions, the multiobjective optimization procedure was formulated as finding, for each different finger mechanism size parameterized by $L_{1}$, the optimal values of link lengths $a_{2}$ and $a_{3}$ corresponding to the largest workspace size, measured in terms of $\alpha_{w}$, achieving a performance of $\mathrm{GII} \leqslant \mathrm{GII}_{\text {target }}$ and respecting the following constraints:

1. A minimum stiffness of $5 \mathrm{~N} / \mathrm{mm}$ at the end effector;

2. A continuous force at the end effector of $2.5 \mathrm{~N}$, with a single stage gear-reduction unit and a maximum torque request of $30 \mathrm{~N} \mathrm{~mm}$; and

3. $L_{1}<150 \mathrm{~mm}$, in order to avoid the interference between the device and the arm.

The above requirements correspond to performance values commonly adopted in haptic literature to achieve a realistic rendering of forces and confirmed by psychophysics studies [27]. The constraint of a single stage gear-reduction unit was motivated by the need for guaranteeing a high level of backdrivability of the system, while the limitation on the maximum torque for the actuators was motivated by the need for keeping a low value for the total weight of the motorization group. Moreover the range of variation of $L_{1}$ was limited by the average dimensions of the human forearm.

The optimization procedure was based on an iterative process. For each finger mechanism and for each value of $L_{1}$, the following steps were followed:

1. Define a starting guess value for $\alpha_{W}$;

2. Find the optimal dimensions of $a_{2}$ and $a_{3}$, through a minimization simplex algorithm, that produce the minimum value for GII over the workspace spanned with the angle $\alpha_{W}$; and

3 If $\left|\mathrm{GII}_{\min }-\mathrm{GII}_{\text {target }}\right|>\epsilon$ :

- If $\mathrm{GII}_{\min }$ is lower than $\mathrm{GII}_{\text {target}}$, increase $\alpha_{W}$;

- If $\mathrm{GII}_{\min }$ is higher than $\mathrm{GII}_{\text {target }}$, decrease $\alpha_{W}$; and

- Update the value of $\alpha_{W}$ with the current one and continue from Step 2,

else finish.

4.4.2 Results. As a final solution of this analysis, a series of curves were obtained corresponding to different optimal kinematic dimensioning of $a_{2}, a_{3}$, expressed in terms of $L_{1}$.

The index GII determines the fidelity of the haptic device in replicating forces and simulating arbitrary mechanical impedances: In fact both static and dynamic properties, such as stiffness and mass of the device, are mediated through the kinematic performance. Moreover the simulation of collisions with virtual walls, requiring high impulsive forces, may be distorted by the saturation of actuators along bad-conditioned directions. According to the performance and previous designs already conducted with this methodology [24,28], the reference values for GII have been chosen in order to guarantee that the force performance along different directions in different points of the workspace differ only by a multiplicative factor lower than 5 or 10 .

The curve plotted in Fig. 9 shows the analytical relationship expressing the size of the largest workspace with $\mathrm{GII}=\mathrm{GII}_{\text {target }}$, parametrized through the maximum achievable wrist angular range $\alpha_{W}(y$ axis), that can be covered with mechanisms of different sizes, parametrized through the distance $L_{1}$ ( $x$ axis). Equivalently the curves represent the maximum angular ranges that can be achieved for $\mathrm{GII}=5$ and $\mathrm{GII}=10$, both for the index and thumb mechanisms.

The results show that behavior of the thumb mechanism is more critical then the index one, because the thumb finger for its greater mobility can reach endpoint positions much closer to the singularity datum plane $\Pi_{1}$. In particular it worths noting that, for the thumb mechanism, $\alpha_{W}>0$ is admitted only for $L_{1 t}>80 \mathrm{~mm}$ with $\mathrm{GII}=5$ and $L_{1 t}>56 \mathrm{~mm}$ with $\mathrm{GII}=10$; for smaller distances the mechanism is not able to track the fingertip workspace, maintaining the required values of GII over the workspace. The thumb mobility is always restricted to the range $\alpha_{W}<25 \mathrm{deg}$ with GII $=5$ and to $\alpha_{W}<50$ deg with $\mathrm{GII}=10$.

On the other side there are no particular constraints to the design of the index mechanism, since the full mobility of wrist $\left(\alpha_{W}=126 \mathrm{deg}\right)$ can be reached for GII $=10$ and $L_{1 i} \geqslant 120 \mathrm{~mm}$.

Together with $\alpha_{w}$, also the other two indexes of performance, RT and MS, were computed for each value of $L_{1}$.

Figures 10 and 11 show the values of the required torques at the actuators and the mechanical stiffness at the end effector, respectively, for the index and the thumb mechanisms. In this case, regular increases of $L_{1}$ worsen the performance values.

As far as the size of actuators, the torque request to the actuators is almost doubled when the mechanism is displaced along the 


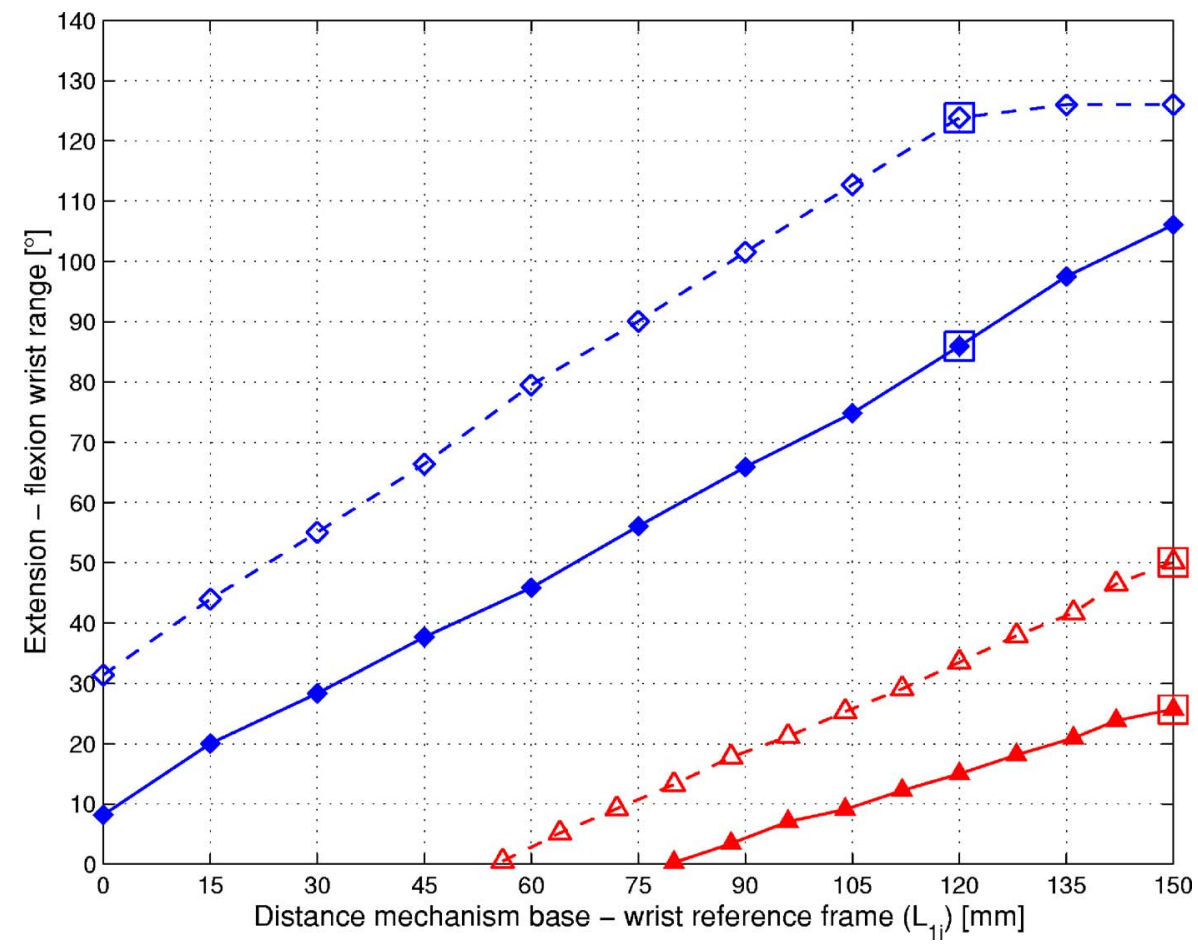

Fig. 9 The workspace $\alpha_{W}$ as a function of $L_{1 i}$ for thumb (gray line with triangle markers) and index (black line with diamond markers) fingers for different values of GII $=5$ (solid line with filled markers) and GII=10 (dashed line with unfilled markers)

forearm (increasing values of $L_{1}$ ). The torque request on the thumb mechanism is slightly lower than on the index one, because the thumb mechanism is characterized by a smaller arm length between the points where forces are applied and motors are posi- tioned, due to the different lengths of human thumb and index fingers, as it is shown in Fig. 8. This property applies as well for values of mechanical stiffness.

From the analysis of torque values required to exert $1 \mathrm{~N}$ along

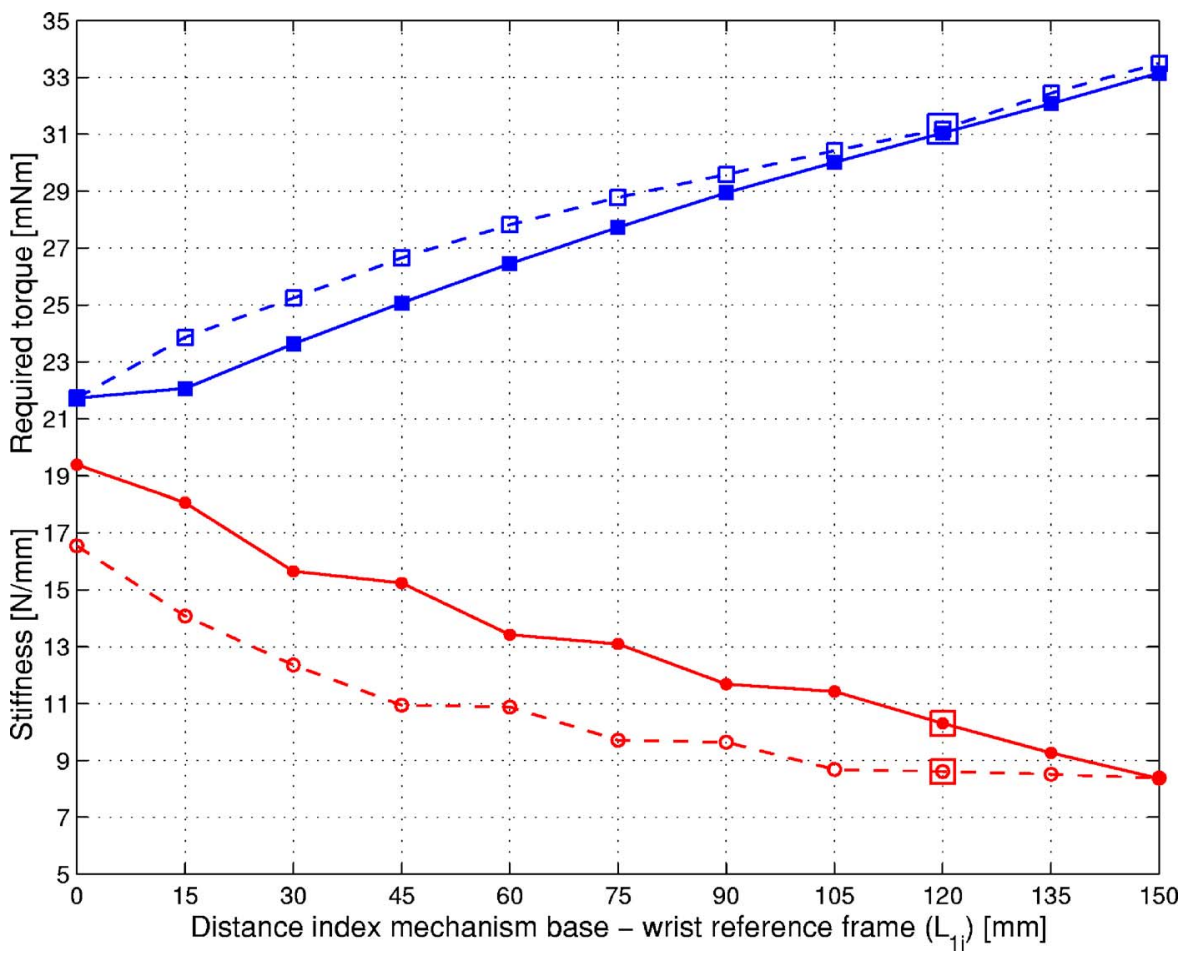

Fig. 10 Stiffness (gray line with circle markers) and maximum torque (black line with square markers) for the index finger for different values of $\mathrm{Gll}=5$ (solid line with filled markers) and GII=10 (dashed line with unfilled markers) 


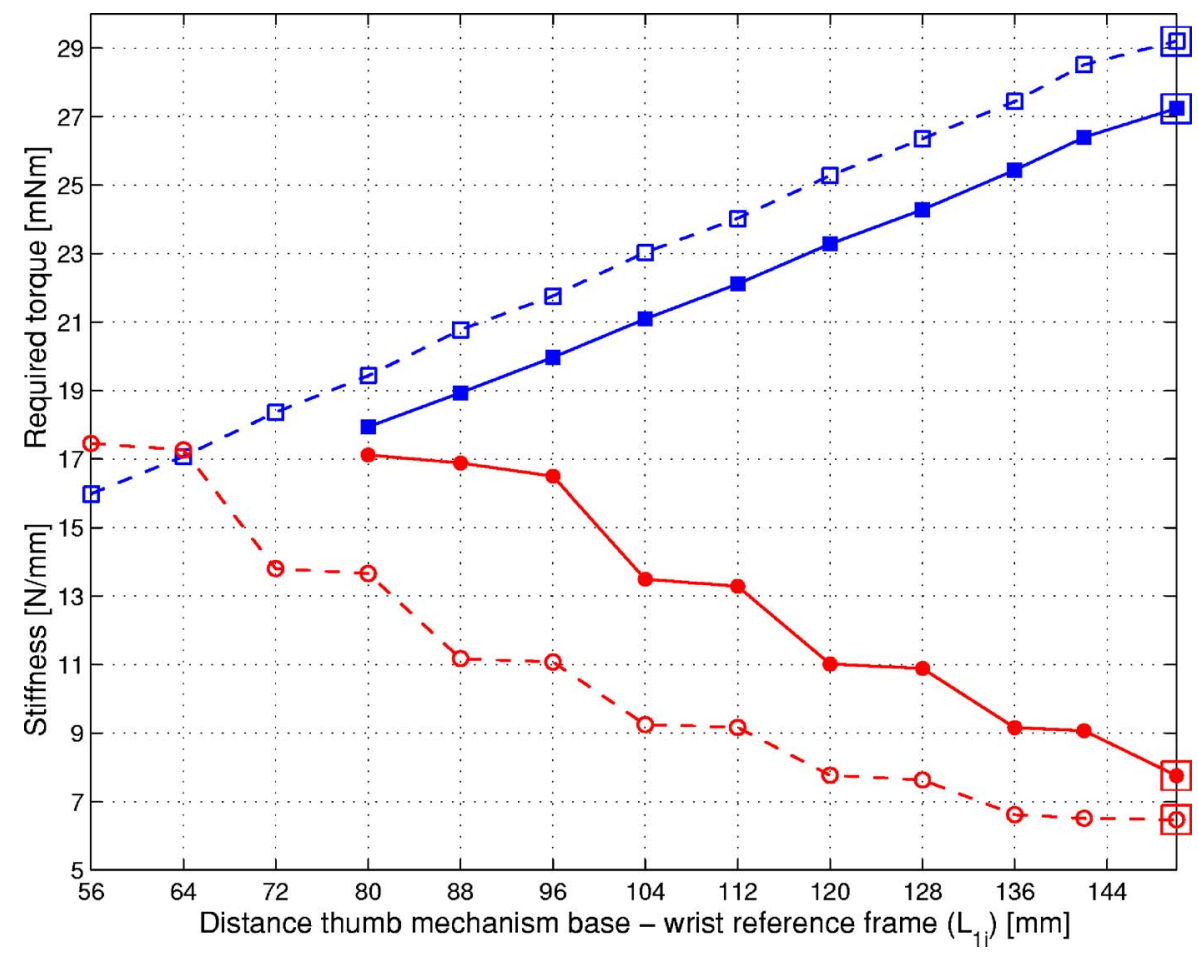

Fig. 11 Stiffness (gray line with circle markers) and maximum torque (black line with square markers) for the thumb finger for different values of Gll=5 (solid line with filled markers) and GII =10 (dashed line with unfilled markers)

any arbitrary direction with the index and thumb fingers, reported in Figs. 10 and 11, we can conclude that to reach the force specifications at the end effector is sufficient to adopt a motor delivering a continuous torque up to $30 \times 2.5 \mathrm{~N} \mathrm{~mm}$, that can be easily accomplished with a single stage reduction unit as by specifications. On the other side, stiffness performance is not critical and can be guaranteed for all admissible dimensions.

\section{Discussion}

The selected optimal values for the two solutions are outlighted in the plots of Figs. 9-11 by a bigger square marker encircling them. For the limb mechanism of the thumb, the base link should be positioned at the furthest admissible distance $L_{1}$ from the fingertip, to guarantee at least a workspace of $\alpha_{w}=25 \mathrm{deg}$ with GII $=5$ and no singularities. As to the index mechanism, it can be instead positioned at a closer distance $L_{1}$. The optimal value for $L_{1}$ was selected in order to achieve a performance of perceived stiffness and required torque similar to the one of the thumb mechanism, but achieving the largest possible workspace. From Fig. 9, it can be easily seen how the optimal solution $\left(L_{1}=120 \mathrm{~mm}\right)$ is located at the knee of the workspace curve for $\mathrm{GII}=10$, where full mobility of the hand is achieved, and bigger values of $L_{1}$ do not gain additional workspace than required.

On the basis of the results of this analysis, the following parameters were chosen for the final configuration

$$
\begin{gathered}
a_{2} / a_{3}=2 / 3 \\
L_{1 i}=-120 \mathrm{~mm} \\
L_{1 t}=-150 \mathrm{~mm}
\end{gathered}
$$

The following performance are achieved at the level of the end effector, that are well beyond the required minimum force:

- Continuous force: $4 \mathrm{~N}$ (index) and $4.4 \mathrm{~N}$ (thumb);

- Max force: $24.9 \mathrm{~N}$ (index) and 27.2 N (thumb);
- Workspace size $\boldsymbol{\alpha}_{\mathbf{w}}$ for $\mathbf{G I I}=\mathbf{5}$ : $85 \mathrm{deg}$ (index) and $15 \mathrm{deg}$ (thumb); and

- Workspace size $\boldsymbol{\alpha}_{\mathrm{w}}$ for $\mathbf{G I I = 1 0 :} 128 \mathrm{deg}$ (index) and $35 \mathrm{deg}$ (thumb).

The prototype of the system is shown in Fig. 12, where it is possible to observe the compactness of the adopted design solution and the optimal fit with the hand anthropometric dimensions. The reachable workspace of each mechanism is $6319 \mathrm{~cm}^{3}$, with a minimum mechanical stiffness in the worst case of $5.9 \mathrm{~N} / \mathrm{mm}$, an average perceived mass at the end effector of $129 \mathrm{~g}$, with a maximum of $508 \mathrm{~g}$ in the points closer to singularities. The function-

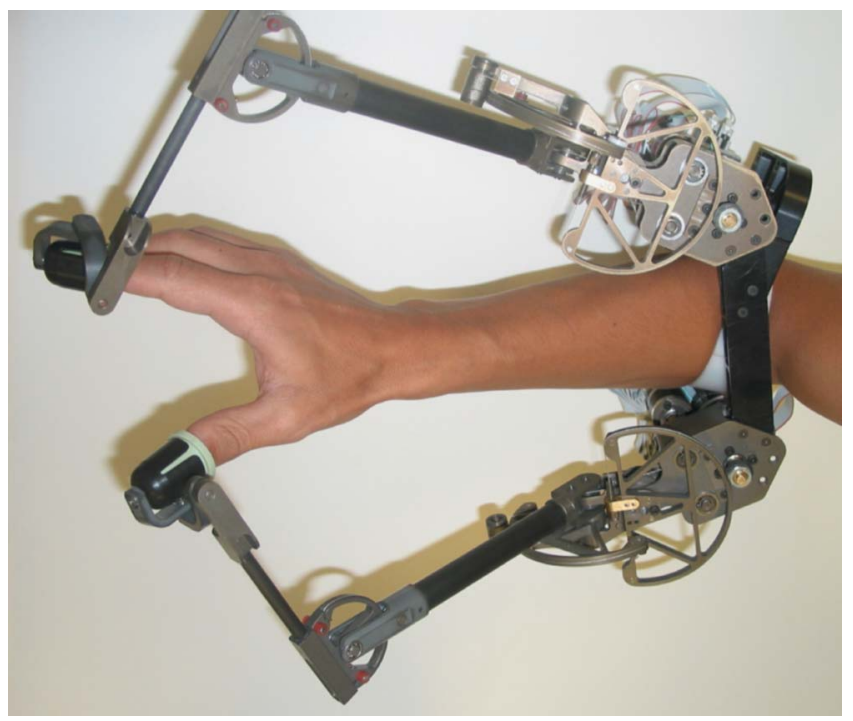

Fig. 12 The prototype of the system 
ality of the device in terms of mobility and level of performance was confirmed by an evaluation of the device carried out in the framework of a research project, where it was used for the interaction with digital reconstructions of sculptures [29].

\section{Conclusions}

This paper has presented the design and the kinematic study of a new portable haptic interface for the thumb and index fingers of the hand. The device is composed of two limbs, actuated through a bilateral tendon transmission, whose main features have been analyzed throughout the paper. An analytical characterization of the performance of this system, in terms of minimum required torque at actuators, maximum reachable workspace, and mechanical stiffness at the end effector, global kinematic isotropy over the workspace has also been presented, outlining the effect and the role played by singularities in the performance of the device. A geometric interpretation of singularities based on screw theory was also introduced to interpret and explain how some hand postures and movements are associated more with a performance worsening.

The paper pointed out how in this case study the combined analysis of hand and mechanism kinematics is needed to completely evaluate the mechanism performance and to define the optimal value of its kinematic parameters. A new original integrated approach for the dimensioning of the system was presented based on a multiobjective optimization.

The results of this paper were used to build a prototype of a new portable haptic interface with two contact points. The final system constitutes a highly innovative haptic interface with respect to the existing state of art, since it allows to achieve a natural two contact points interaction in virtual environments, including two fingers shape exploration, precise manipulation, and grasping of objects. The system has been already successfully employed in a series of exhibitions in European museums, where visitors where allowed to physically touch digital models of sculptures [14], and a usability study conducted on the field confirmed a positive judgment from the users [29]. As future usage, the system remains particularly suitable for the study of sensorymotor control mechanisms in human manipulation and occupational therapy in functional rehabilitation of patients with motor impairments.

\section{References}

[1] Steger, R., Lin, K., Adelstein, B. D., and Kazerooni, H., 2005, "Design of a Passively Balanced Spatial Linkage Haptic Interface," J. Mech. Des., 126, p. 984-991.

[2] Buchholz, B., and Armstrong, T. J., 1995, "A Kinematic Model of the Human Hand to Evaluate its Prehensile Capabilities," J. Biomech., 25(2), pp. 149162.

[3] Jansson, G., and Monaci, L., 2006, "Identification of Real Objects Under Conditions Similar to Those in Haptic Displays: Providing Spatially Distributed Information at the Contact Areas is More Important Than Increasing the Number of Areas," J. Virtual Reality, 9(4), pp. 243-249.

[4] Frisoli, A., Wu, S. L., Ruffaldi, E., and Bergamasco, M., 2005, "Evaluation of Multipoint Contact Interfaces in Haptic Perception of Shapes," Multi-Point Interaction with Real and Virtual Objects, K. Salisbury, F. Barbagli, and D. Prattichizzo, eds., Springer Tracts in Advanced Robotics, Springer, New York, Vol. 18.

[5] Bergamasco, M., Ruffaldi, E., Avizzano, C. A., Frisoli, A., and Marcheschi, S. 2006, "Design and Validation of a Complete Haptic System for Manipulative Tasks," Adv. Rob., 20(3), pp. 367-389.

[6] Butterfa, J., Grebenstein, M., Liu, H., and Hirzinger, G., 2001, "Dlr-hand ii: Next Generation of a Dextrous Robot Hand," Proceedings IEEE International Conference on Robotics and Automation, Seoul, Korea, 1, pp. 109-114.

[7] Morrell, J. B., and Salisbury, J. K., 1996, "Performance Measurements for
Robotic Actuators," Proceeding of the ASME Dynamics Systems and Control Division, Atlanta, GA, November, 58, pp. 531-537.

[8] Ryew, S., and Choi, H., 2001, "Double Active Universal Joint (Dauj): Robotic Joint Mechanism for Humanlike Motions," IEEE Trans. Rob. Autom., 17(3), pp. 290-300.

[9] Turki, L., and Coiffet, P., 1995, "On Grasp Synthesis and Planning of Multifingered Robot Hands for a Telemanipulation Task," Proceedings 4th IEEE International Workshop on Robot and Human Communication, Tokyo, Japan, July, pp. 141-146.

[10] Bergamasco, M., 1996, "Force Replication to the Human Operator: The Development of Arm and Hand Exoskeletons as Haptic Interfaces," In G. Giralt and G. Hirzinger, eds., Robotics Research, The 7th International Symposium, Munich, Germany, Springer, New York, pp. 173-182.

[11] Shields, B. L., Main, J. A., Peterson, S. W., and Strauss, A. M., 1997, "An Anthropomorphic Hand Exoskeleton to Prevent Astronaut Hand Fatigue During Extravehicular Activities," IEEE Trans. Syst. Man Cybern., Part A. Syst. Humans, 27(5), pp. 668-673.

[12] Nakagawara, S., Kajimoto, H., Kawakami, N., Tachi, S., and Kawabuchi, I., 2005, "An Encounter-Type Multi-Fingered Master Hand Using Circuitous Joints," Proceedings of the IEEE ICRA International Conference on Robotics \& Automation, Barcelona, Spain, April, pp. 2667-2672.

[13] Springer, S. L., and Ferrier, N. J., 2002, "Design and Control of a ForceReflecting Haptic Interface for Teleoperational Grasping," J. Mech. Des., 124, p. $277-283$.

[14] Bergamasco, M., Jansson, G., and Frisoli, A., 2003, “A New Option for the Visually Impaired to Experience 3D Art at Museums: Manual Exploration of Virtual Copies," Visual Impairment Research, 5, pp. 1-12.

[15] Frisoli, A., Simoncini, F., and Bergamasco, M., 2002, "Mechanical Design of a Haptic Interface for the Hand," Proceedings 2002 ASME International DETC-27th Biennial Mechanisms and Robotics Conference, Montreal, CA, September.

[16] Rocchi, F., Frisoli, A., Marcheschi, S., Dettori, A., Salsedo, F., and Bergamasco, M., 2005, "A New Force-Feedback Arm Exoskeleton for Haptic Interaction in Virtual Environments," Proceedings of WorldHaptics, 1st Joint EuroHaptics Conference and IEEE Symposium on Haptic Interfaces for Virtual Environments and Teleoperator Systems, Pisa, Italy, March, pp. 195-201.

[17] Marcheschi, S., Frisoli, A., Avizzano, C. A., and Bergamasco, M., 2005, “A Method for Modeling and Controlling Complex Tendon Transmissions in Haptic Interfaces," Proceedings of the IEEE ICRA International Conference on Robotics \& Automation, Barcelona, Spain, April, pp. 1773-1778.

[18] Frisoli, A., Simoncini, F., Salsedo, F., and Bergamasco, M., 2006, "Haptic Interface Device," ep1629949 app n. ep20040020621 20040831, March.

[19] Denavit, J., and Hartenberg, R. S., 1995, "A Kinematic Notation for LowerPair Mechanisms Based on Matrices," ASME J. Appl. Mech., 22, pp. 215221.

[20] Kapandji, I. A., 1982, The Physiology of the Joints, Churchill Livingstone, Oxford, UK.

[21] Wickens, C. D., Lee, J. D., Liu, Y., and Gordon-Becker, S., 2004, Introduction to Human Factors Engineering, Prentice Hall, Englewood Cliffs, NJ.

[22] Gallardo, J., Rico, J. M., Frisoli, A., Checcacci, D., and Bergamasco, M. 2003, "Dynamics of Parallel Manipulators by Means of Screw Theory," Mech. Mach. Theory, 38(11), pp. 1113-1131.

[23] Hunt, K. H., 1978, Kinematic Geometry of Mechanisms, Oxford University Press, Oxford, UK.

[24] Stocco, L., Saculdean, S. E., and Sassani, F., 1998, "Fast Constrained Global Minimax Optimization of Robot Parameters," Robotica, 16, pp. 595-605.

[25] Prisco, G. M., and Bergamasco, M., 1997, "Dynamic Modelling of a Class of Tendon Driven Manipulators," Proceedings ICAR, Monterey, CA, July, pp. 893-899.

[26] Bergamasco, M., and Frisoli, A., 2003, "Experimental Identification and Evaluation of Performance of a 2DOF Haptic Display," Proceedings of the IEEE ICRA Conference, Taipei, Taiwan, September, pp. 3260-3265.

[27] Hayward, V., and Astley, O., 1998, "Performance Measures for Haptic Interfaces," Robotics Research: Proceedings 7th International Symposium, G. Giralt and G. Hirzinger, eds., Munich, Germany, Springer, New York, pp. 195 207.

[28] Prisco, G. M., Frisoli, A., Salsedo, F., and Bergamasco, M., 1999, "A Novel Tendon Driven 5-Bar Linkage With a Large Isotropic Workspace," Proceedings ASME IMECE '99, International Mechanical Engineering Conference Exhibition, Nashville, TN

[29] Bergamasco, M., Frisoli, A., Jansson, G., and Loscos, C., 2005, "Evaluation of the Pure-Form Haptic Displays Used for Exploration of Works of Art at Museums," Proceedings of WorldHaptics, 1st Joint EuroHaptics Conference and IEEE Symposium on Haptic Interfaces for Virtual Environments and Teleoperator Systems, Pisa, Italy, March (CDRom) 